\title{
Y Chromosome and HIV DNA Detection in Vaginal Swabs as Biomarkers of Semen and HIV Exposure in Women
}

\author{
Kerri J. Penrose, MS, * Barbra A. Richardson, PhD, $\dagger$ Guillaume Besson, PhD, * \\ Charlene S. Dezzutti, PhD, ** Betsy C. Herold, MD, \& Salim S. Abdool Karim, PhD, $\mathbb{1}$ \\ John W. Mellors, MD, * and Urvi M. Parikh, PhD,* \\ and on behalf of the MTN Biomedical Sciences Working Group and the HPTN 035 Protocol Team
}

\begin{abstract}
Background: The inability to quantify sexual exposure to HIV limits the power of HIV prevention trials of vaccines, microbicides, and preexposure prophylaxis in women. We investigated the detection of HIV-1 and Y chromosomal (Yc) DNA in vaginal swabs from 83 participants in the HPTN 035 microbicide trial as biomarkers of HIV exposure and unprotected sexual activity.

Methods: One hundred forty-three vaginal swabs from 85 women were evaluated for the presence of Yc DNA (Quantifiler Duo DNA quantification kit; Applied Biosystems) and total HIV-1 DNA (single-copy in-house quantitative polymerase chain reaction assay). Y DNA detection was paired with self-reported behavioral data with regard to recent coitus $(\leq 1$ week before collection) and condom usage ( $100 \%$ vs. $<100 \%$ compliance).

Results: Yc DNA was detected in $62(43 \%)$ of 143 swabs. For the 126 visits at which both behavioral data and swabs were collected, Yc DNA was significantly more frequent in women reporting less than $100 \%$ condom usage (odds ratio, 10.69; 95\% confidence interval, 2.27-50.32; $P=0.003$ ). Notably, 27 (33\%) of 83 swabs from women reporting $100 \%$ condom usage were positive for Yc DNA. HIV DNA was only detected in swabs collected postseroconversion.

Conclusions: The use of Yc DNA in HIV prevention trials could reliably identify subgroups of women who have unprotected sexual activity and could provide valuable exposure-based estimates of efficacy.
\end{abstract}

From the *University of Pittsburgh, Pittsburgh, PA; †University of Washington, Seattle, WA; \$Magee-Womens Research Institute, Pittsburgh, PA; $\S$ Albert Einstein College of Medicine, New York, NY; and 9 Centre for the AIDS Programme of Research in South Africa, Durban, South Africa

Financial support: This work was supported by the Bill and Melinda Gates Foundation (Grant 48293) and the Microbicide Trials Network, which is cofunded by the National Institute of Allergy and Infectious Diseases (Grant UM1 AI068633), the National Institute of Child Health and Development, and the National Institute of Mental Health, all of the National Institutes of Health.

Potential conflicts of interest: J.W.M. is a consultant for Gilead Sciences and owns shares of RFS Pharmaceuticals. No other authors have reported conflicts.

Previous Presentation: This work was partially presented at the 20th Conference on Retroviruses and Opportunistic Infections in Atlanta, Georgia; March 3-7, 2013

Correspondence: Urvi M. Parikh, PhD, Division of Infectious Diseases, Department of Medicine, University of Pittsburgh, S817 Scaife Hall, 3550 Terrace St, Pittsburgh, PA 15261. E-mail: ump3@pitt.edu.

Received for publication May 1, 2014, and accepted August 6, 2014.

DOI: 10.1097/OLQ.0000000000000191

Copyright (C) 2014 American Sexually Transmitted Diseases Association All rights reserved.
A major limitation of current HIV prevention trial design is the lack of an accurate and sensitive means to measure exposure to HIV. ${ }^{1,2}$ The evaluation of risk behavior using selfreported condom use and frequency of coitus can be unreliable because of miscommunication between interviewer and interviewee, lack of understanding of the questions asked, reporting behavior according to perceived expectations, inability to recall experience, and blatant misreporting. ${ }^{3,4}$ In addition, self-report of condom use frequency does not capture risk associated with improper condom use or accidental condom breakage. Despite greater than $80 \%$ reported condom use in VOICE, FEM-PrEP, and the TDF2 study, pregnancy and HIV incidence rates remained higher than expected. ${ }^{5-7}$ A cross-sectional study of 910 women in Zimbabwe found that only $52 \%$ of participants who tested positive for prostate-specific antigen (PSA) in vaginal swabs reported unprotected sex during the previous 2 days. Audio computer-assisted self-interview technology did not generate significantly different responses about unprotected intercourse compared with face-to-face interview. ${ }^{8}$

The detection of semen could provide an unbiased measure of unprotected sex in participants of HIV prevention trials. However, the limited sensitivity of the most commonly used biomarker, PSA, makes it impractical for use in clinical studies where swab collection could occur hours to weeks after intercourse. Levels of PSA decline 10 -fold by 3 hours postexposure and are undetectable by 24 to 48 hours postexposure. ${ }^{9-11}$ Rapid stain identification of human semen that detects the presence of semenogelin has also been used in trials to indicate that a woman has been exposed to ejaculate in the previous 48 hours, but it is 10 -fold less sensitive than quantitative polymerase chain reaction (PCR) methods to detect Y chromosomal (Yc) DNA. ${ }^{12,13}$ Depite heterogeneity in both initial deposit of Yc DNA and rate of decline of Yc DNA signal, Yc has the advantage of detectability up to 15 postcoital days from self-collected vaginal swabs without impact from menses on the rate of decay. ${ }^{14,15} \mathrm{Y}$ chromosome DNA is not detected in women using condoms correctly, as demonstrated by a study that showed that only 5 of 56 women had positive Yc DNA results after condom use after a 14-day abstinence period, and the 5 detections were associated with receptive oral sex and digital penetration. ${ }^{16,17}$

The detection of HIV in genital samples from HIV-negative women could more directly assess HIV infection risk. In a study of cervical dysplasia in US women, HIV-1 env and gag glycoproteins were identified in cervicovaginal lavage samples from women who were confirmed to be HIV negative by serology. ${ }^{18}$ 
However, partner HIV status was not known, and the linkage of env and gag detection with future seroconversion was not verified. HIV-1 viral RNA and proviral HIV-1 DNA sequences can be detected in seminal plasma and nonspermatazoal mononuclear cells in HIV-infected men throughout successful long-term highly active antiretroviral therapy, whereas plasma HIV-1 viral RNA levels remain undetectable. ${ }^{19-22}$ The detection of HIV DNA in genital samples from HIV-negative women has not been studied as a marker of HIV infection risk.

We therefore examined the frequency of Yc DNA and HIV DNA detection in vaginal swabs collected in HIV seroconverters (both preseroconversion and postseroconversion) and nonseroconverters from the HPTN 035 study, using highly sensitive quantitative PCR assays with detection limits of a single copy.

\section{MATERIALS AND METHODS}

\section{Study Population}

HPTN 035 was a phase II/IIb safety and effectiveness study of the vaginal microbicides BufferGel and 0.5\% PRO2000/5 gel for the prevention of HIV-1 infection in women, conducted from February 2005 through September 2008 (NCT00074425). All participants provided informed consent for swab collection and future testing. The population demographic characteristics, protocol, and trial results are described elsewhere. ${ }^{23}$ Starting in 2008, vaginal swab specimens were collected from participants during each quarterly pelvic examination by applying a Dacron swab to the posterior fornix of the vagina until the tip was saturated with fluid, then placing the swab in a cryovial containing $400 \mu \mathrm{L}$ of phosphate-buffered saline. The cryovials were stored at $-80^{\circ} \mathrm{C}$ at the sites and shipped to the MTN Network Laboratory after the primary study results were available. The current study evaluated a case-control subset of swabs collected from women at sites in Zimbabwe (Harare and Chitungwiza), South Africa (Hlabisa and Durban), and Malawi (Blantyre and Lilongwe) at a 1:3 ratio (seroconverters/nonseroconverters). Swabs from seroconverters were collected both preseroconversion and postseroconversion. Swabs from participants postseroconversion were collected a median of 21 days after detection of seroconversion (range, 5-124 days). Seroconverters were not taking antiretroviral therapy at the time of swab collection. The operator performing the assays was blinded to the subgroup to which the participant belonged.

\section{Nucleic Acid Extraction}

Swabs were processed to isolate the cell pellet as described previously. ${ }^{24}$ Total nucleic acid was extracted by incubating the vaginal swab cell pellet in $2 \mathrm{mg} / \mathrm{mL}$ Proteinase K solution (Applied Biosystems) for 30 minutes at $55^{\circ} \mathrm{C}$. Guanidinium isothiocyanate (Sigma) and glycogen (Roche) were added to final concentrations of $4.58 \mathrm{M}$ and $0.47 \mathrm{mg} / \mathrm{mL}$, respectively, and incubated at room temperature for 30 minutes. Nucleic acids were precipitated by centrifugation at $15,000 \times g$ in the presence of a nearly equal volume of isopropanol. Nucleic acid pellets were washed repetitively with $70 \%$ ethanol and air dried before suspending in $5 \mathrm{mM}$ Tris, $\mathrm{pH}$ 8. One third of the sample was used as template in the Quantifiler Duo assay, one third was used for testing HIV-1 DNA, and one third was stored.

\section{Detection of HIV-1 DNA}

HIV-1 DNA was detected using a modified version of the single copy assay with primers targeted to a conserved region in the integrase gene (iSCA). ${ }^{25}$ Briefly, $10 \mu \mathrm{L}$ of extracted DNA was diluted with $20 \mu \mathrm{L}$ of $5 \mathrm{mM}$ Tris $\mathrm{pH} 8$ and run in triplicate in a reaction containing $1 \times$ Roche LightCyler 480 probes master mix, $400 \mathrm{nM}$ of primers iSCA-F (5'-TTT GGA AAG GAC CAG CCA A-3') and iSCA-r (5'-CCT GCC ATC TGT TTT CCA-3') and $200 \mathrm{nM}$ Taqman probe (5'-6FAM AAA GGT GAA GGG GCA GTA GTA ATA C BHQ_1-3'). DNA was amplified at $95^{\circ} \mathrm{C}$ for 5 minutes followed by 45 cycles of $95^{\circ} \mathrm{C}$ for 15 seconds and $60^{\circ} \mathrm{C}$ for 1 minute on a LightCycler 480 (Roche). This assay can detect HIV-1 DNA at a single copy per well, as verified by Poisson distribution statistics in limiting dilution experiments of purified HIV-1 DNA target in mock swab cell pellets prepared using A431 cells spiked with a known quantity of purified HIV-1 DNA and human semen. False-positive results were observed in 0 of 111 reactions using Tris buffer as a no template control. Each sample was run in triplicate and considered "undetectable" if all 3 reactions had no amplification; "detected, quantifiable" if at least 2 of 3 reactions had 1 or more copies detected per reaction; and "detected, not quantifiable" if only 1 of 3 reactions had 1 or more copies detected.

\section{Detection of Yc DNA}

Y chromosome and total human DNA was detected using the commercially available Quantifiler Duo kit (Applied Biosystems). Reactions were prepared according to the manufacturer's guidelines with the modification of increasing the total number of PCR cycles from 40 to 55 to allow late amplification ( $>35$ cycles) to reach the plateau phase of PCR, and to observe if any cases of nonspecific amplification occurred after 40 cycles. Despite these adjustments, all positive wells had cycle threshold values of 40 cycles or below, consistent with the kit manufacturer's guidelines. Wells with cycle threshold values of 40 to 41 that had exponential amplification were included as positive only if the calculated copy number was 1 copy or above. No cases of false amplification between 41 and 55 cycles occurred. The assay can detect Yc DNA at a single copy per well, as verified by Poisson distribution statistics in limiting dilution experiments of semen in mock swab cell pellets. False-positive Yc DNA was observed in 0 of 135 reactions using Tris buffer as a no template control and 0 of 65 reactions using A431 female epithelial cell pellets as a negative control. Positive detection using dilutions of semen was obtained in 89 of 89 wells with a predicted copy number of 2 or higher. Additional sensitivity and specificity information can be found in the Quantifiler Duo user's manual. Each test sample was run in replicates of 5 and considered "undetectable" if all 5 reactions had no amplification; "detected, quantifiable" if at least 3 of 5 reactions had 1 or more copies detected per reaction; and "detected, not quantifiable" if only 1 or 2 reactions had at least 1 copy detected per reaction. Total Human DNA, measured through the RPPH1 target as part of the Quantifiler Duo kit, was used to calculate approximate total cell numbers in each swab cell pellet and quantified according to kit manufacturer's instructions.

\section{Behavioral Data Collection and Analysis}

Participants were queried on gel and condom use during the last coital act and during all coital acts in the last 7 days at each quarterly visit, and data were collected by participant selfreport. ${ }^{23}$ Detected, but not quantifiable Yc DNA samples were excluded from the analysis of Yc DNA detection with self-reported condom use because of the inferior quality of these samples.

\section{Statistical Analysis}

SPSS Version 20 (IBM Corp.) was used for all analyses. Conditional logistic regression was used to compare the odds of being a seroconverter among those with detectable Yc DNA and those with no detectable Yc DNA. The association between Yc DNA detection (vs. no detection) and reported sexual activity was assessed using generalized estimating equation models with a binary link, robust errors, and independent correlation structure. 


\section{RESULTS}

\section{Swab Collection and Cell Recovery}

In the HPTN 035 study, 3524 vaginal swab specimens were collected from 2031 women from the African sites. A randomly selected subset of 110 swabs from 65 women who remained HIV negative throughout the study (controls) and 33 swabs from 18 women who seroconverted postenrollment (cases) were evaluated for the presence of HIV and Yc DNA (Fig. 1). Of 33 swabs from seroconverters, 10 were collected, whereas the women were still HIV negative (before seroconversion) and 23 were collected after the participant was confirmed as having seroconverted. Cell recovery from swabs as assessed through total human DNA detection was a median of $3.0 \times 10^{5}$ cells/swab (interquartile range, $1.4 \times 10^{5}$ to $6.1 \times 10^{6}$ cells/swab). Receiver operating characteristic curve analysis demonstrated no minimal cell pellet size requirement for the detection of Yc DNA. The minimum cell number needed for HIV DNA recovery could not be determined because of small sample size (data not shown).

\section{HIV-1 DNA Detection}

HIV-1 DNA was only detected in 10 of 23 swabs collected postseroconversion. Five had quantifiable HIV-1 DNA with a median of 141 copies/sample and a range of 55 to 1593 copies/ sample from swabs that were collected postseroconversion, and 5 had HIV-1 DNA that was detected but not quantifiable (Table 1). HIV-1 DNA could not be detected in the 10 samples collected before seroconversion or in the 110 samples from women who remained HIV-1 negative throughout the study.

\section{Yc DNA Detection}

All 143 swabs were evaluated for the presence of Yc DNA as a measure of unprotected sexual activity. Y chromosome DNA was detected in 62 of 143 (43\%) swabs. Forty-four of 62 samples had quantifiable Yc DNA, with a median of 425 copies/sample and a range of 20 to 11926 copies/sample. Y chromosome DNA was detected but not quantifiable in 18 of $62(29 \%)$ samples (Table 2). Using conditional logistic regression with serostatus as the outcome, no significant differences in Yc DNA detection were found between the HIV seroconverter cases (5/33 [15\%]) and HIV nonseroconverter controls $(39 / 110[35 \%])$ in samples that had quantifiable Yc DNA $(P=0.1)$. This difference remained nonsignificant when samples collected before seroconversion were included as cases (odds ratio [OR], 0.48; $P=0.3$ ). There was no correlation between Yc and HIV-1 DNA detection in the sample set.

\section{Association of Yc DNA Detection With Self-Reported Condom Use}

Participant responses to frequency of coitus and condom use were also collected at 126 of the 143 visits at which a swab sample was collected. There were $27(21 \%)$ reports of no vaginal sex in the week before sample collection, $83(66 \%)$ reports of vaginal sex in the past week with $100 \%$ condom use, and $16(13 \%)$

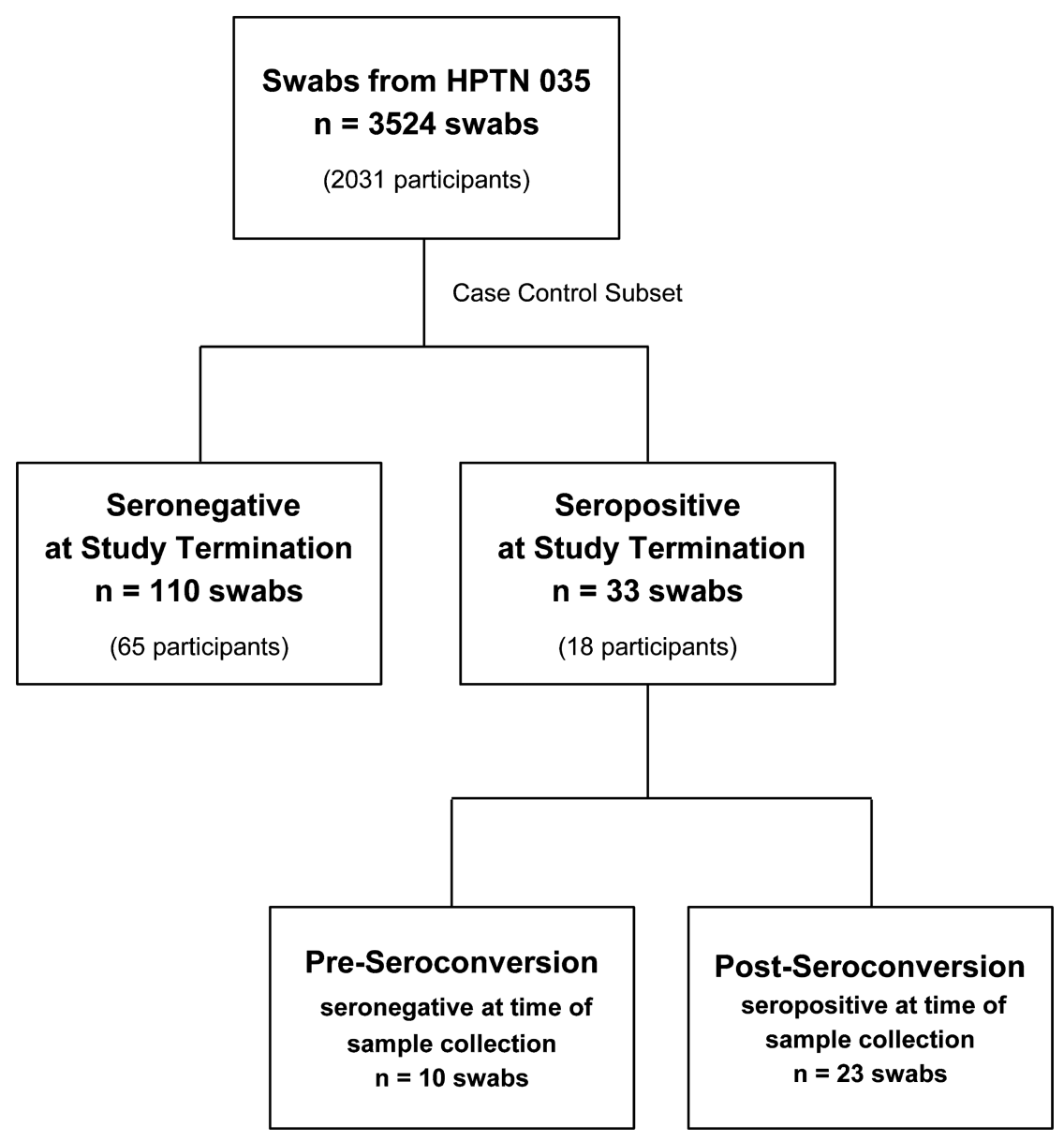

Figure 1. Study design. 
TABLE 1. Detection of HIV-1 DNA in a Subset of Vaginal Swabs From HPTN 035

\begin{tabular}{|c|c|c|c|c|}
\hline \multirow[b]{2}{*}{ HIV-1 DNA* } & \multirow{2}{*}{$\begin{array}{c}\text { Seronegative }^{\dagger} \\
(\mathrm{n}=110), \text { n }(\%)\end{array}$} & \multirow{2}{*}{$\begin{array}{l}\text { Preseroconversion } \\
\quad(n=10), n(\%)\end{array}$} & \multicolumn{2}{|c|}{ Postseroconversion $(n=23)$} \\
\hline & & & n $(\%)$ & Median (range) copies/sample \\
\hline Undetectable & $110(100)$ & $10(100)$ & $13(50)$ & - \\
\hline Detected, quantifiable & 0 & 0 & $5(25)$ & $141(55-1593)$ \\
\hline Detected, not quantifiable & 0 & 0 & $5(25)$ & - \\
\hline
\end{tabular}

*Each sample was run in triplicate and considered "undetectable" if all 3 reactions had no amplification; "detected, quantifiable" if at least $2 / 3$ reactions had 1 or more copies detected per reaction; and "detected, not quantifiable" if only 1 of 3 reactions had 1 or more copies detected.

Swab sample collected from participant who remained seronegative at study termination.

${ }^{\ddagger}$ Swab sample collected from participant who was seronegative at time of sample collection, but seropositive at study termination.

reports of vaginal sex in the past week with less than $100 \%$ condom use. Excluding samples that were not quantifiable, the proportion of participants with Yc DNA increased as reported condom use decreased. Only 4 (15\%) of 27 samples from participants reporting no coitus had Yc DNA (median, 98 copies), compared with 27 (33\%) of 83 samples with Yc DNA (median, 518 copies) from participants reporting vaginal sex in the past week with $100 \%$ condom use and $9(56 \%)$ of 16 samples from participants with Yc DNA (median, 568 copies) reporting vaginal sex in the past week with less than $100 \%$ condom use. The proportion of samples with Yc DNA was significantly higher (OR, $10.69 ; P=0.003)$ among those participants with inconsistent condom use compared with those reporting no coitus in the past week (Table 3). Reporting of feminine hygiene practices did not correlate with Yc DNA detection (data not shown).

\section{DISCUSSION}

The advent of new, highly sensitive quantitative PCR technologies for nucleic acid detection and quantification in genital specimens enabled us to evaluate the feasibility of using HIV and Yc DNA detection as a biomarker for risk behavior in HIV prevention clinical trials. We quantified HIV and Yc DNA from the same vaginal swab and stratified the results against behavioral data on condom use from women both preseroconversion and postseroconversion and from women who never seroconverted in the trial period. The operator performing the assays was blinded as to the subgroups to which the participants belonged.

Despite having an assay with sensitivity down to a single copy of HIV-1 DNA per PCR reaction, we did not find evidence of HIV-1 in specimens from seronegative women, including from those women who eventually seroconverted. The inability to detect seminal viral DNA (from an infected male partner) in a vaginal swab sample could have been influenced by sample quality, timing of swab sample collection from last coital act, and lack of frequent exposure to HIV. Furthermore, $\mathrm{CD}^{+}$lymphocytes only comprise
$2 \%$ of the total cell number in an average semen sample and only $0.1 \%$ of $\mathrm{CD}^{+} \mathrm{T}$ cells carry provirus in an HIV-infected individual. The number of absolute $\mathrm{CD} 4^{+}$lymphocytes in semen can also depend on the health of the individual and decrease dramatically in viremic patients. ${ }^{26}$ Of note, the maximum number of Yc DNA copies from any swab in our study was 1000 copies/well, with the majority (134/143 [94\%]) having Yc copies of 100 or less, meaning that only a small fraction of semen was being detected. Because so little of Yc DNA is being recovered from an ejaculate, it is very unlikely that rare HIV-infected cells would have been detected.

We also did not observe a difference in frequency of Yc DNA detection in seroconverters compared with nonseroconverters ( $15 \%$ vs. $35 \%)$, and the number of copies of Yc detected did not predict risk of seroconversion. Walsh et al. ${ }^{27}$ showed that there was a significant difference in levels of PSA and sperm counts with different types of risk exposures such as condom breakage, but a similar analysis using Yc DNA has not been done. There are several explanations for Yc DNA not predicting seroconversion including variation in donor sperm count, variable time between last coitus and sample collection, noncoital exposure (e.g. digital), and limited sampling of seroconverters. Larger observations over longer periods may reveal an association of Yc DNA exposure and risk of HIV or other sexually transmitted infections.

Our data do provide further evidence that Yc chromosomal DNA detection serves as a reliable biomarker to monitor sexual activity. ${ }^{28}$ A significantly higher proportion of women $(P=0.003)$ reporting unprotected sex in the past week had detectable Yc DNA compared with women reporting no vaginal sex in past week. Interestingly, $33 \%$ of women reporting $100 \%$ condom use had detectable Yc DNA, with copy numbers at similar levels to those women reporting less than $100 \%$ condom use (518 vs. 568 copies), suggesting that condom usage was overreported in this study or that exposure occurred before the use of a condom. The behavioral questionnaire was administered through face-to-face interviews, which may contribute to inconsistent reporting particularly for

TABLE 2. Detection of Yc DNA in a subset of vaginal swabs from HPTN 035

\begin{tabular}{lccrrr}
\hline & \multicolumn{2}{c}{ Seronegative $^{\dagger}(\mathbf{n}=\mathbf{1 1 0})$} & & \multicolumn{2}{c}{ Seropositive $^{\ddagger}(\mathbf{n}=\mathbf{3 3})$} \\
\cline { 2 - 3 } \cline { 5 - 6 } Yc DNA* & $\mathbf{n}(\mathbf{\%})$ & Median (range) copies/sample & & $\mathbf{n}(\mathbf{\%})$ & Median (range) copies/sample \\
\hline Undetectable & $62(56)$ & - & $19(58)$ & - \\
Detected, quantifiable & $39(35)$ & $413(16-7003)$ & & $5(15)$ & $626(74-11926)$ \\
Detected, not quantifiable & $9(8)$ & - & $9(27)$ & -
\end{tabular}

*Each sample was run in replicates of 5 and considered "undetectable" if all 5 reactions had no amplification; "detected, quantifiable" if at least 3 of 5 reactions had 1 or more copies detected per reaction; and "detected, not quantifiable" if only 1 or 2 reactions had at least 1 copy detected per reaction.

Swab sample collected from participant who remained seronegative at study termination.

${ }^{+}$Swab sample collected from participant who was seropositive at study termination. Ten samples were collected preseroconversion, and 23 samples were collected postseroconversion. 
TABLE 3. Correlation of YC DNA with Self-Reported Condom Usage

\begin{tabular}{|c|c|c|c|c|}
\hline & \multicolumn{2}{|r|}{ Yc DNA } & \multicolumn{2}{|c|}{ Univariate Analysis } \\
\hline & $\begin{array}{l}\text { Not Detected, } \\
\text { n (\%) }\end{array}$ & $\begin{array}{c}\text { Detected, Quantifiable, } \\
\text { n }(\%)^{*}\end{array}$ & OR $(95 \%$ CI $)$ & $\boldsymbol{P}$ \\
\hline No vaginal sex in past week $(n=27)$ & $19(70)$ & $4(15)$ & 1 & - \\
\hline Vaginal sex in past week with $100 \%$ condom use $(n=83)$ & $44(53)$ & $27(33)$ & $2.92(0.93-9.13)$ & 0.066 \\
\hline Vaginal sex in past week with $<100 \%$ condom use $(n=16)$ & $4(25)$ & $9(56)$ & $10.69(2.27-50.32)$ & 0.003 \\
\hline
\end{tabular}

CI indicates confidence interval

Samples that were detected but not quantifiable were excluded from the analysis.

sensitive topics. ${ }^{29}$ Our data show promise for the use of Yc DNA as an objective measure for condom use. Further study is needed to determine if level of risk or type of risk behavior can be correlated with number of Yc copies detected.

One limitation of this study is that testing for HIV and Yc DNA was done retrospectively on stored swabs from quarterly sampling, where timing of swab collection after coitus and the HIV infection status of the male partner was not known. Testing a larger number of preseroconversion swabs or swabs from serodiscordant couples could provide further insight into the feasibility of using HIV or Yc DNA as a biomarker. Self-collected samples could provide the best timing for detecting HIV exposure, but would rely on the ability and willingness of participants to collect high-quality samples. More frequent sample collection could provide risk information to statisticians for refined secondary analysis of clinical trial data in populations of highest risk. Modifying the assay for HIV detection to include HIV-1 RNA or total nucleic acid detection may improve sensitivity.

In summary, we demonstrated that by using highly sensitive quantitative PCR assays, Yc DNA and total HIV-1 DNA can be detected down to a single copy in vaginal swab samples. Y chromosome DNA detection is more frequent among women reporting less than $100 \%$ condom use with coitus but can also be detected in a third of women reporting $100 \%$ condom usage. These results suggest that Yc DNA detection in vaginal fluids could refine assessments of HIV risk and efficacy of preventive strategies.

\section{REFERENCES}

1. Mauck CK, Doncel GF. Biomarkers of semen in the vagina: applications in clinical trials of contraception and prevention of sexually transmitted pathogens including HIV. Contraception 2007; 75:407-419.

2. Mauck CK. Biomarkers for evaluating vaginal microbicides and contraceptives: Discovery and early validation. Sex Transm Dis 2009; 36(3 suppl):S73-S75.

3. Anglewicz P, Gourvenec D, Halldorsdottir I, et al. The effect of interview method on self-reported sexual behavior and perceptions of community norms in Botswana. AIDS Behav 2013; 17:674-687.

4. Pool R, Montgomery CM, Morar NS, et al. Assessing the accuracy of adherence and sexual behaviour data in the MDP301 vaginal microbicides trial using a mixed methods and triangulation model. PLoS One 2010; 5:e11632.

5. Marrazzo J, Ramjee G, Nair G, et al. Pre-exposure prophylaxis for HIV in women: Daily oral tenofovir, oral tenofovir/emtricitabine or vaginal tenofovir gel in the VOICE study (MTN 003). 20th Conference on Retroviruses and Opportunistic Infections, Atlanta, GA; 2013.

6. Thigpen MC, Kebaabetswe PM, Paxton LA, et al. Antiretroviral preexposure prophylaxis for heterosexual HIV transmission in Botswana. N Engl J Med 2012; 367:423-434.

7. Van Damme L, Corneli A, Ahmed K, et al. Preexposure prophylaxis for HIV infection among African women. N Engl J Med 2012; 367 : $411-422$.
8. Minnis AM, Steiner MJ, Gallo MF, et al. Biomarker validation of reports of recent sexual activity: Results of a randomized controlled study in Zimbabwe. Am J Epidemiol 2009; 170:918-924.

9. Bahamondes L, Diaz J, Marchi NM, et al. Prostate-specific antigen in vaginal fluid after exposure to known amounts of semen and after condom use: Comparison of self-collected and nurse-collected samples. Hum Reprod 2008; 23:2444-2451.

10. Jamshidi R, Penman-Aguilar A, Wiener J, et al. Detection of two biological markers of intercourse: Prostate-specific antigen and Y-chromosomal DNA. Contraception 2013; 88:749-757.

11. Macaluso M, Lawson L, Akers R, et al. Prostate-specific antigen in vaginal fluid as a biologic marker of condom failure. Contraception 1999;59:195-201.

12. Abbott SA, Friedland BA, Sarna A, et al. An evaluation of methods to improve the reporting of adherence in a placebo gel trial in Andhra Pradesh, India. AIDS Behav 2013; 17:2222-2236.

13. Simson Oechsle C, Haddad S, Sgueglia JB, et al. Screening biological stains with qPCR versus lateral flow immunochromatographic test strips: A quantitative comparison using analytical figures of merit. J Forensic Sci 2014; 59:199-207.

14. Brotman RM, Melendez JH, Smith TD, et al. Effect of menses on clearance of Y-chromosome in vaginal fluid: Implications for a biomarker of recent sexual activity. Sex Transm Dis 2010; 37:1-4

15. Zenilman JM, Yuenger J, Galai N, et al. Polymerase chain reaction detection of Y chromosome sequences in vaginal fluid: Preliminary studies of a potential biomarker for sexual behavior. Sex Transm Dis $2005 ; 32: 90-94$.

16. Melendez JH, Giles JA, Yuenger JD, et al. Detection and quantification of Y-chromosomal sequences by real-time PCR using the LightCycler system. Sex Transm Dis 2007; 34:617-619.

17. Ghanem KG, Melendez JH, McNeil-Solis C, et al. Condom use and vaginal Y-chromosome detection: The specificity of a potential biomarker. Sex Transm Dis 2007; 34:620-623.

18. Basu J, Romney SL, Angeletti RH, et al. Human immunodeficiency virus (HIV) antigens and RNA in HIV-seronegative women with cervical intraepithelial neoplasia. AIDS Res Hum Retroviruses 2009; $25: 249-259$

19. Mermin JH, Holodniy M, Katzenstein DA, et al. Detection of human immunodeficiency virus DNA and RNA in semen by the polymerase chain reaction. J Infect Dis 1991; 164:769-772.

20. Ball JK, Curran R, Irving WL, et al. HIV-1 in semen: Determination of proviral and viral titres compared to blood, and quantification of semen leukocyte populations. J Med Virol 1999; 59:356-363.

21. Zhang H, Dornadula G, Beumont M, et al. Human immunodeficiency virus type 1 in the semen of men receiving highly active antiretroviral therapy. N Engl J Med 1998; 339:1803-1809.

22. Lambert-Niclot S, Tubiana R, Beaudoux C, et al. Detection of HIV-1 RNA in seminal plasma samples from treated patients with undetectable HIV-1 RNA in blood plasma on a 2002-2011 survey. AIDS 2012; 26:971-975.

23. Abdool Karim SS, Richardson BA, Ramjee G, et al. Safety and effectiveness of BufferGel and $0.5 \%$ PRO2000 gel for the prevention of HIV infection in women. AIDS 2011;25:957-966.

24. Dezzutti CS, Richardson BA, Marrazzo JM, et al. Mucosal Escherichia coli bactericidal activity and immune mediators are associated with HIV-1 seroconversion in women participating in the HPTN 035 trial. J Infect Dis 2012; 206:1931-1935. 
25. Cillo AR, Krishnan A, Mitsuyasu RT, et al. Plasma viremia and cellular HIV-1 DNA persist despite autologous hematopoietic stem cell transplantation for HIV-related lymphoma. J Acquir Immune Defic Syndr 2013; 63:438-441.

26. Politch JA, Mayer KH, Anderson DJ. Depletion of $\mathrm{CD}^{+} \mathrm{T}$ cells in semen during HIV infection and their restoration following antiretroviral therapy. J Acquir Immune Defic Syndr 2009; 50:283-289.

27. Walsh TL, Frezieres RG, Peacock K, et al. Use of prostate-specific antigen (PSA) to measure semen exposure resulting from male condom failures: Implications for contraceptive efficacy and the prevention of sexually transmitted disease. Contraception 2003; 67:139-150.

28. Jadack RA, Yuenger J, Ghanem KG, et al. Polymerase chain reaction detection of Y-chromosome sequences in vaginal fluid of women accessing a sexually transmitted disease clinic. Sex Transm Dis 2006; 33:22-25.

29. Phillips AE, Gomez GB, Boily MC, et al. A systematic review and meta-analysis of quantitative interviewing tools to investigate selfreported HIV and STI associated behaviours in low- and middleincome countries. Int J Epidemiol. 2010;39(6):1541-1555. 